\title{
Radiographic Imaging Analysis after Sling Exercises for Hemiplegic Shoulder Subluxation
}

\author{
Myung-kwon Kim, PT, PhD ${ }^{1)}$, Jae-min Jung, PhD²), Jong-Sung Chang, PT, $\mathrm{PhD}^{3)}$, \\ Su-KYOUNG LEe, PT, MS ${ }^{4)}$ \\ 1) Department of Physical Therapy, Youngsan University \\ 2) Department of Physical Therapy, Daegu University \\ 3) Department of Physical Therapy, Honam University \\ 4) Department of Physical Therapy, Gimhae College: Sambang-Dong, Gimhae-si, Kyeongsangnam-do, \\ Republic of Korea. TEL: +82 10-3581-2628,FAX: +82 55-3366-222,E-mail: holeintwo@naver.com
}

\begin{abstract}
Purpose] The aim of this study was evaluate the effect of a sling exercise on shoulder subluxation in stroke patients. [Subjects] The subjects of this study were thirty-four stroke patients with subluxation. They were randomly selected and divided into two groups. [Methods] The subject group $(\mathrm{n}=17)$ performed the sling exercise with traditional therapeutic exercise. The control group $(\mathrm{n}=17)$ performed only traditional therapeutic exercise. [Results] The results indicate that improvement of corrected vertical distance (cVD), and the ratio of the oblique distance of the affected and unaffected sides (rOD) was greater in the experimental group than in the control group. [Conclusion] We suggest that the sling exercise is effective at reducing the severity of hemiplegic shoulder subluxation in post-stroke patients.

Key words: Radiographic imaging, Sling exercise, Shoulder subluxation
\end{abstract}

(This article was submitted May 14, 2012, and was accepted Jun. 13, 2012)

\section{INTRODUCTION}

Spasticity and paralysis are common symptoms which patients with stroke often exhibit in the upper extremitities, and they can cause problems with shoulder subluxation, adhesive capsulitis, impingement syndrome and rotator cuff injury ${ }^{1,2)}$. In shoulder subluxation, the space between the humerus and the acromion of the shoulder blade is expanded and the mechanism and alignment are changed as gravity drags down the relaxed limb ${ }^{3,4)}$. This initial dragging pain can lead to temporal or long-term nerve damage ${ }^{5)}$. Further, enlarged neurovascular and musculoskeletal tissue around the shoulder joint may result in etiology of other symptoms such as limited dynamic range, pain, brachial nerve injury, and reflexive sympathetic nerve system atrophy ${ }^{6,7)}$. Shoulder subluxation, caused by hemiplegia after stroke, can impede the rehabilitation of a patient as it can cause impairment of daily life and movement. Therefore, it is important in the rehabilitation of patients with hemiplegic stroke to treat shoulder subluxation $^{8,9)}$. Shoulder subluxation is generally treated by use assister which can substitute the paralyzed intermuscular and electric stimulus ${ }^{6)}$.

Sling exercise therapy is a treatment method which uses active-exercise treatment with a sling aiming to decrease neuromuscular disorder ${ }^{10)}$. This method is used to improve the health of normal subjects and to treat nervous system disorders, musculoskeletal system disorders, and sports injuries in both, young children and the elderly. Sling exercise therapy utilizes the helping hands theory, hanging point change, stabilization exercise principle, open/close chain exercise principle and sensorimotor control. This treatment method can benefit patients with central nervous disorders through improving control of counteractions against gravity, which affect patient during exercise, and reflect controlling. This research investigated the effect of sling exercise therapy, which is been used to strengthen the proprioceptive neuromuscular system, on treatment of shoulder subluxation of patients with hemiplegic stroke.

\section{SUBJECTS AND METHODS}

Thirty-four patients those who had been diagnosed as having stroke by CT and MRI analysis were the subjects of this study. Twenty-five of them of them were judged to have had infarct and post-stroke, nine were judged to have had hemorrhagic strokes (Table 1). All of the subjects were for more than nine months, and were mostly neurogically recovered. None had had fracture, trauma or operation on the affected side. All they had no case history of diabetes or other diseases which can damage peripheral nerves. Those whose result of manual muscle test (MMT) result were zero or negligible were excluded from the research. The participants were given sufficient explanation about the research process and they submitted their written consent showing that they had agreed to participate in the study voluntarily. The participants were divided into an experimental group (17) and a control group (17).

Both the experimental and control groups received tradi- 
Table 1. The general characteristics of the subjects

\begin{tabular}{ccc}
\hline & EG & CG \\
\hline Sex(M/F) & $4 / 13$ & $5 / 12$ \\
Type of stroke & $6 / 11$ & $5 / 12$ \\
(Hemorrhage/Infarction) & & \\
Age(years) & $64.7 \pm 12.3$ & $62.2 \pm 15.3$ \\
K-NIHSS & $11.30 \pm 1.06$ & $11.20 \pm 2.20$ \\
K-MMSE & $21.20 \pm 2.44$ & $21.00 \pm 3.88$ \\
Time since onset(months) & $21 \pm 3.2$ & $16.8 \pm 5.7$ \\
Affected side (Rt/Lt) & $7 / 11$ & $6 / 9$ \\
\hline
\end{tabular}

$(\mathrm{Mean} \pm \mathrm{SD})$

tional physical therapy once a day for half an hour, five times a week and the research period lasted out for eight weeks. Sling exercise therapy for half an hour a day was additionally carried out by the experimental group. Traditional physical therapy consisted of range of motion exercise and stretching for ten minutes, weight bearing exercise for ten minutes, and range of motion exercise for ten minutes, lasting for thirty minutes in total. In detail, the range of motion and stretching implemented for the joints the hemiplegic arm and leg to prevent contracture. In the weight bearing exercise, loads were placed on the hemiplegic arm and leg to stimulate their muscles, and movement was performed. All exercises were conducted by a which were the same for both the therapist, and the participants were familiar with exercises and principles experimental and control groups. For the sling exercise, we used a portable stand therapy-master (TM Plus Extra, Therapymaster AS, Norway) which is equipped with a portable stand. The focus was on strengthening the rotator cuff muscles, in particular, and the deltoid muscle which is related to the stability of the arm in shoulder subluxation patients. The exercise program began with from active exercise and progressed to resistance exercise. In the sling exercise, abduction/adduction, and internal rotation/ external rotation after fixing their elbow and wrist joints were performed. All exercises were isometric, with subjects trying to hold position against resistance provided by an examiner

The modified method suggested by Brooke et al. ${ }^{9)}$ was employed to measure the level of subluxation radiographs. We identified three points, the inner lowest part of the acromion, center of the humerus head, and the center of the glenoid fossa, and measured the vertical distance (VD), and horizontal distance (HD), and the length of the straight line connecting the center of the humerus head and the acromion which we defined as the oblique distance (OD). Each distance was divided by the upper and lower longest distance to the glenoid fossa to calculate, $\mathrm{cVD}$ (corrected vertical distance), cHD (corrected horizontal distance) and cOD (corrected oblique distance). We also calculated $\mathrm{rVD}$, the ratio of both vertical distances of the affected and unaffected sides, and rOD, the ratio of both oblique distances of the affected and unaffected sides (Fig. 1). To analyze the collected data, SPSS ver. 17.0 for Windows was used. To test the homogeneity of the experimental and control groups,

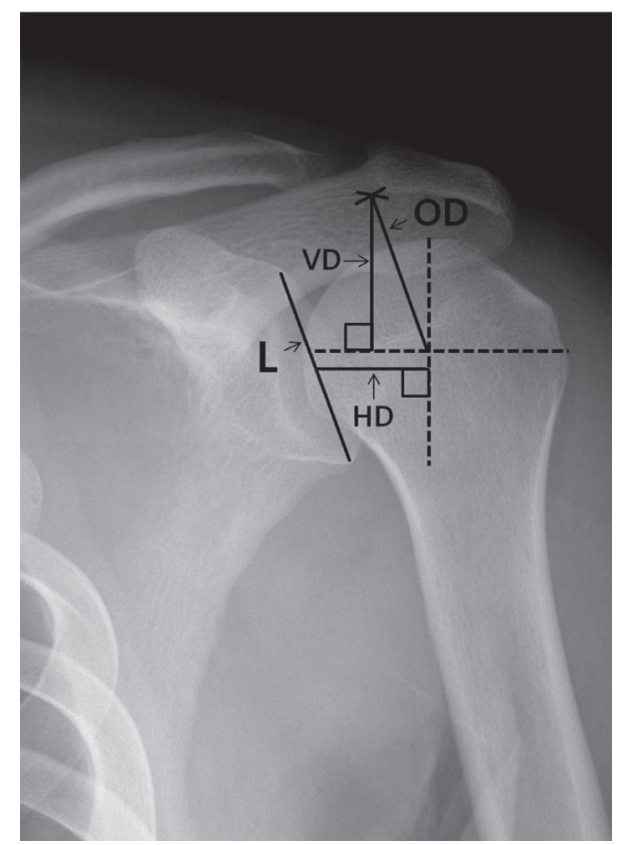

Fig. 1. Measurement of VD, HD, OD between the glenoid fossa and humerus VD: Vertical distance; HD: Horizontal distance; OD: Oblique distance

the $\chi^{2}$-test was employed for gender and affected side, which are categorical data, and the independent $t$ test was employed to test continuous data including age and symptom. The average and standard deviation of each group was calculated and the paired $t$ test was used to investigate the within group changes before and after the experiment. The independent $t$ test was used to test the differences between the groups before and after the experiment. Significance was accept for values of $\mathrm{p}<0.05$.

\section{RESULTS}

After the interventions, cVD, rVD, rOD showed significant improvements after the sling exercise in the experimental group, and cVD showed a significant improvement in the control group. In the comparison of the experimental and control groups, $\mathrm{cVD}$, and rOD after the sling exercise in the experimental group showed significantly greater improvements than those of the control group (Table 2).

\section{DISCUSSION}

It is difficult for patients with stroke to recover the function of the upper extremity and this can cause limitations in daily life which can lead to exclusion from society. Hence, it is important to establish an effective recovery method for the upper extremity. Cailliet ${ }^{11)}$ reported that for shoulder subluxation in a with stroke, the scapula was directed exactly up to lateral hence for humerus head to direct to down, it needs to move to side at the same time. Further, in 
Table 2. Outcome measures before and after sling the intervention

\begin{tabular}{llll}
\hline & & \multicolumn{1}{c}{ EG } & CG \\
\hline \multirow{2}{*}{$\mathrm{cVD}^{*}$} & pre & $2.90 \pm 0.66$ & $2.88 \pm 0.69$ \\
& post & $2.57 \pm 0.75^{*}$ & $2.41 \pm 0.68^{*}$ \\
$\mathrm{rVD}$ & pre & $1.20 \pm 0.27$ & $1.15 \pm 0.17$ \\
& post & $0.88 \pm 0.21^{*}$ & $0.92 \pm 0.19$ \\
$\mathrm{cHD}$ & pre & $2.62 \pm 0.39$ & $2.61 \pm 0.45$ \\
& post & $2.74 \pm 0.43$ & $2.66 \pm 0.44$ \\
$\mathrm{cOD}$ & pre & $3.43 \pm 0.88$ & $3.38 \pm 0.67$ \\
& post & $3.36 \pm 0.56$ & $3.19 \pm 0.54$ \\
$\mathrm{rOD} *$ & pre & $1.16 \pm 0.53$ & $1.12 \pm 0.28$ \\
& post & $1.02 \pm 0.16^{*}$ & $1.11 \pm 0.12$ \\
\hline
\end{tabular}

$(\mathrm{Mean} \pm \mathrm{SD}){ }^{*} \mathrm{p}<0.05$. EG: Experimental group; CG: Control group. cVD: Corrected vertical distance; rVD: ratio of the vertical distances of the affected and unaffected sides; cHD: corrected horizontal distance; cOD: corrected oblique distance; rOD: ratio of the oblique distances of the affected and unaffected sides

preventing subluxation through a such locking mechanism as supraspinatus and deltoid, tension of such organ as articular capsule, the subluxation occurs indirection of front and low as humerus head moves due to weakened shoulder muscle in case of patient with stroke. Stroke patients with stroke with subluxation have longer vertical and, oblique distances at the shoulder joint than normal. Therefore, strengthening the weakened supraspinatus and deltoid muscle can be seen as a fundamental treatment method for subluxation. In this research, and abduction/adduction, internal rotation/ external rotation were performed by using a sling exercise for hemiplegic shoulder subluxation to strengthen the rotator cuff and deltoid muscles. The sling exercise was conducted isometrically to strengthen the rotator cuff muscle and to improve the stability of the upper arm. As the following results stability exercise increase function of deep muscle and range of motion ${ }^{12)}$, I think sling exercise using as a kind of stability exercise could get a good effect to stability muscle such a rotator cuff muscle.

The sling exercise would have stimulated the neuromuscular system and reactivated muscles with weakness and pain caused by subluxation, through high intensity isometric contraction $^{13)}$. Lephart and Henry ${ }^{14)}$ said that dynamic stabilization of a single joint was needed for recovery of neuromuscular control. It can be gained by co-contraction of agonist and antagonist and neuromuscular training of a single joint with a sling.

Brooke's method ${ }^{9)}$, which is a modified version of a quantified method, induced greater improvement in the experimental group than in the control group after the sling exercise in vertical distance and the ratios of the affected and unaffected sides. This may be because the contact surface between the humerus head and glenoid fossa was broadened as the muscle strengthened. The rotator cuff and deltoid muscles are involved in the stability of the glenohumeral joint was enhanced compared to before the performance of the sling exercise. Huang ${ }^{15)}$ reported that warming up with a sling exercise resulted in equivalent throwing velocity and accuracy that were similar to those reported for the thrower's 10 warm-up method.

The limitation of sling exercises is that they require muscle strength over the second level (Poor grade) in the hemiplegic upper extremity as measured by manual muscle testing, and it is very difficult to utilize this method for patients in the acute phase. However, the sling method is a useful treatment method for preventing hemiplegic shoulder subluxation after acute the phase.

\section{REFERENCES}

1) Turner-Stokes L, Jackson D: Shoulder pain after stroke: a review of the evidence base to inform the development of an integrated care pathway. Clin Rehabil, 2002, 16: 276-298. [Medline] [CrossRef]

2) Zorowitz RD, Gross E, Polinski DM: The stroke survivor. Disabil Rehabil, 2002, 24: 666-679. [Medline] [CrossRef]

3) Dursun E, Dursun N, Ural CE, et al.: Glenohumeral joint subluxation and reflex sympathetic dystrophy in hemiplegic patients. Arch Phys Med Rehabil, 2000, 81: 944-946. [Medline] [CrossRef]

4) Hanger HC, Whitewood P, Brown G, et al.: A randomized controlled trial of strapping to prevent post-stroke shoulder pain. Clin Rehabil, 2000, 14: 370-380. [Medline] [CrossRef]

5) Wang RY, Chan RC, Tsai MW: Functional electrical stimulation on chronic and acute hemiplegic shoulder subluxation. Am J Phys Med Rehabil, 2000, 79: 385-390. [Medline] [CrossRef]

6) Vuagnat H, Chantraine A: Shoulder pain in hemiplegic revisited: contribution of functional electrical stimulation and other therapies. J Rehabil Med, 2003, 35: 49-54. [Medline] [CrossRef]

7) Lo SF, Chen SY, Lin HC, et al.: Arthrographic and clinical findings in patients with hemiplegic shoulder pain. Arch Phys Med Rehabil, 2003, 84: 1786-1791. [Medline] [CrossRef]

8) Delisa JA, Gans BM: Rehabilitation medicine: Principles and practice (2nd ed), Philadephia: 1993, pp 814-815.

9) Brooke MM, de Lateur BJ, Diana-Rigby GC, et al.: Shoulder subluxation in hemiplegia: effects of three different supports. Arch Phys Med Rehabil, 1991, 72: 582-586. [Medline]

10) Kirkesola G: Sling exercise therapy (S-E-T): a total concept for exercise and active treatment of musculoskeletal disorders. Korean J Orthop Manu Ther, 2001, 7: 87-106.

11) Cailliet R: The shoulder in hemiplegia. Philadelphia: FA Davis Company, 1995, pp 63-68.

12) Kisner C, Colby LA: Therapeutic exercise foundations and techniques. Philadelphia: F. A. Davis, 1990, pp325-336.

13) Kirkesola G.: S-E-T advanced level 1-U. The upper body. Seminar workbooks. Norway: S-E-T Kompetanse AS. 2005.

14 Lephart SM, Fu FH: Proprioception and neuromuscular control in joint stability. Human Kinetics, 2000, pp 405-413.

15) Huang JS, Pietrosimone BG, Ingersoll CD: Sling exercise and traditional warm-up have similar effects on the velocity and accuracy of throwing. J Strength Cond Res, 2011, 25: 1673-1679. [Medline] [CrossRef] 